\title{
connaissance du gisement et étude d'impact
}

\author{
par \\ C. Archimbaud \\ Laboratoire Régional des Ponts et Chaussées, Clermont-Ferrand \\ L. Primel \\ L.C.P.C., Paris
}

RESUME - Pour certaines carrières, la législation récente rend obligatoire l'étude d'impact avant ouverture. Les textes imposent que cette étude doit comprendre 5 parties : définition de l'état initial, - effets prévisibles sur l'environnement, - motivations de choix du site, - dispositions pour prévenir, réduire et compenser les effets des nuisances, - remise en état des sols. Les études d'impact actuellement réalisées prennent rarement en compte les caractéristiques géométriques et qualitatives du futur gisement. Cette communication a pour but de montrer qu'une bonne étude du gisement est indispensable à la réalisation d'une étude d'impact plausible; les méthodes de prospection utilisées aussi bien en roches massives, que pour les gisements alluvionnaires, permettent de mettre en évidence les différents paramètres (essentiellement épaisseur et nature de la découverte et du matériau exploitable, contexte hydrogéologique) dont la connaissance est indispensable pour toute prévision du mode d'exploitation du gisement et donc de son influence sur l'environnement, ainsi que pour un phasage réaliste de la remise en état des sols.

\section{INTRODUCTION}

Lorsqu'un exploitant sollicite l'autorisation d'étendre son gisement ou a'en exploiter un nouveau, il doit se référer en France au décret $n^{\circ} 79-1108$ du 20/12/1979, traitant du nouveau régime des autorisations d'ouverture de carrières. Ce texte réglementaire impose de bien prendre en compte les "contraintes"; mais trop souvent ne vient à l'esprit que ce qui concerne l'environnement au sens large : le paysage, le milieu naturel, la protection des nappes, les nuisances dues au bruit et poussières..., en oubliant, ou tout au moins en sous-estimant la contrainte située la plus en amont et qui pourtant est à la base de toutes les autres : le GISEMENT. Pourtant, il faut considérer que l'article 8 du décret est très explicite sur ce point. Nous n'aurons pas la cruauté de rappeler les nombreux exemples de carrières ouvertes dans des gisements "fantômes", le but de cette communication étant de montrer en quoi la connaissance du gisement est une donnée fondamentale pour une prise en compte sérieuse et réaliste de l'environnement.

Quels sont les paramètres qu'il est indispensable de connaitre d'une part pour réaliser une étude d'impact fiable et d'autre part pour organiser 1'exploitation, de façon à réduire ou supprimer les impacts négatifs et respecter les conditions prévues pour la remise en état des sols? Telle est la question posée.

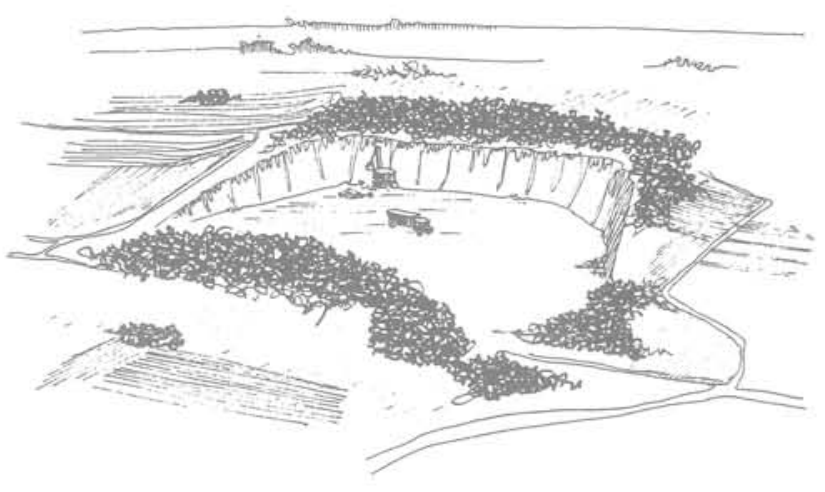

Fig.1 - Utilisation des différents éléments d'un site (topographie, gisement, végétation)en vue de diminuer les contraintes visuelles.

\section{LA DECOUVERTE}

Il est évident que l'exploitant doit connấtre l'épaisseur de la découverte qu'il aura à décaper, ainsi que les variations de cette épaisseur. Economiquement parlant, le matériel employé dépendra directement de ces 
notions d'épaisseur, mais aussi de la nature de la découverte et bien entenđu de la position de la nappe phréatique lorsqu'elle existe. Jusqu'à présent, ces questions n'étaient en gênéral étudiées que dans le cadre d'une gestion économique des terrassements. Le problème du "devenir" de cette découverte était rarement envisagé sérieusement, et c'est là que l'on commence à aborder les problèmes d'environnement :

- le stockage de la découverte suppose, comme nous le verrons par la suite, une bon. ne connaissance du gisement : combien de cas ne connait-on pas où les dépôts ont été fait sur les meilleurs zones d'un site. Ces stockis ne doivent eux-mêmes pas constituer une atteinte à l'environnement, soit par exemple par leur insertion non prévue dans le paysage, soit que leur lessivage ait entrainé une pollution des cours d'eau ou des nappes.

- l'emploi des dépôts de découverte peut au contraire être prévu dès l'étude d'environnement, par exemple, pour la constitution de merlons revégétalisables (et dans ce cas, il faut prévoir une éventuelle sẻparation entre terre végétale et le reste de la découverte) qui serviront de protection contre le bruit, ou contre les poussiēres, voire même d'écran de camouflage pour une meilleure intégration de la carrière dans le site.

- le stockage de la découverte doit enfin être organisé en fonction de ce qui est prévu dans l'étude d'impact pour la remise en état du site au fur et à mesure et en fin d'exploitation. Ces prévisions peuvent aller du simple remodelage jusqu'à la reconstitution de terres agricoles.

\section{LE MATERIAU EXPLOITABLE}

Chacun sait qu'il est très rare qu'une exploitation englobe la totalité du périmètre prévu : il existe toujours dans une extraction des zones abandonnées soit parce que le matériau est d'une cualité médiocre, soit parce que la pollution est trop forte pour que l'exploitation puisse être faite dans des conditions économiques satisfaisantes. En général, ces "délaissées" donnent à la carrière un aspect détestable, alors que la mise en évidence de ces zones lors de l'étude de cisement aurait permis de les insérer dan's le phasage de l'exploitation et de prévoir leur remise en état. De plus, comme il faut de toute façon choisir un emplacement pour les installations, pourquoi ne pas choisir de préférence ces zones inexploitables pour y installer les matériels de concassage, criblage, recomposition, ainsi que les stocks étant entendu que $1^{\prime}$ on assure en même temps leur insertion dans 1'environnement. Dans d'autres gisements, on peut par contre prévoir l'utilisation des zones inexploitables comme moyen de lutte contre les nuisances diverses : bruit des installations et de l'extraction, poussières émises au concassage ou au broyace, front de taille trop visible.

En ce qui concerne les zones exploitées, il est bien entendu indispensable pour réaliser une étude d'impact plausible de bien connaítre les épaisseurs exploitables, leurs variations sur l'ensemble du gisement, c'est-àdire en fait, disposer d'un plan du substratum après exploitation, ainsi çue de données suffisantes sur sa nature, dont dépendront directement les possibilites de remiseen état.

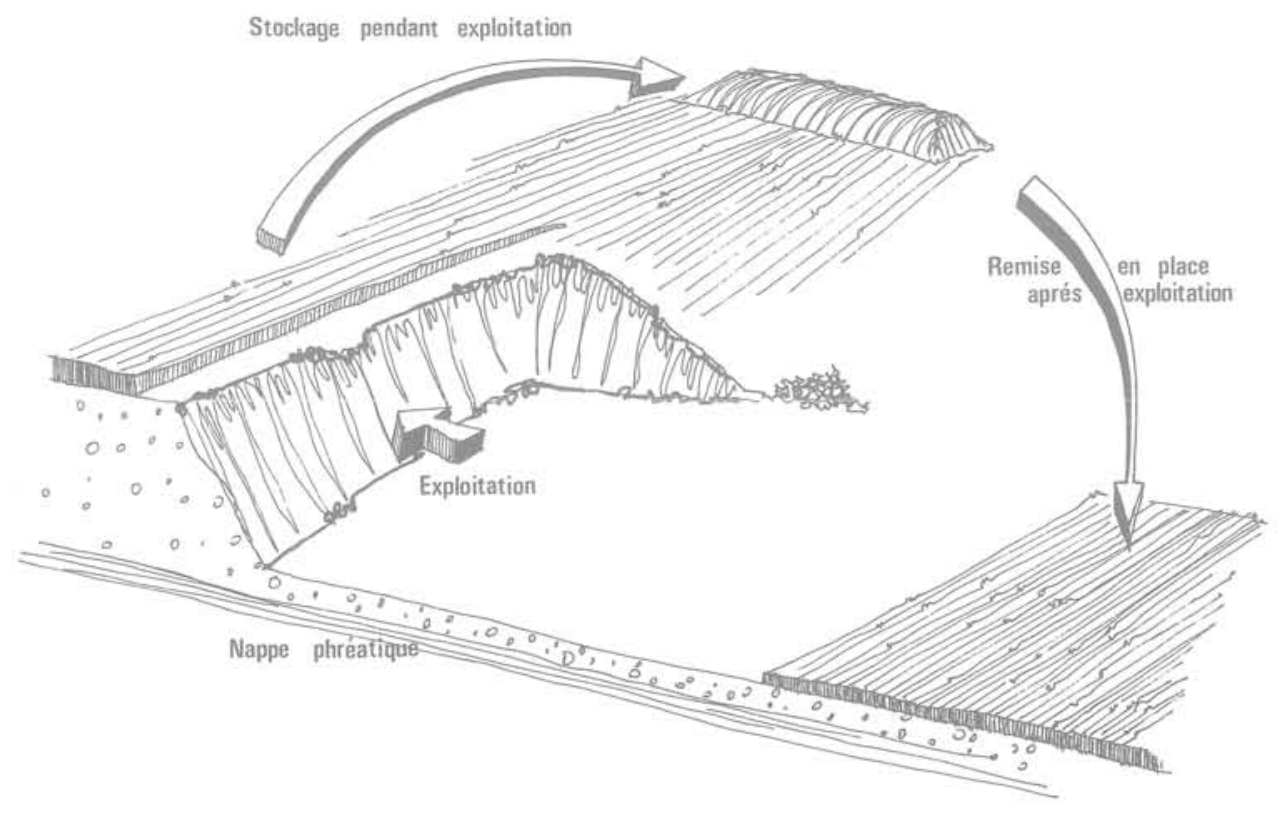

Fig. 2 - Phasage d'exploitation : mouvement des terres de découverte 


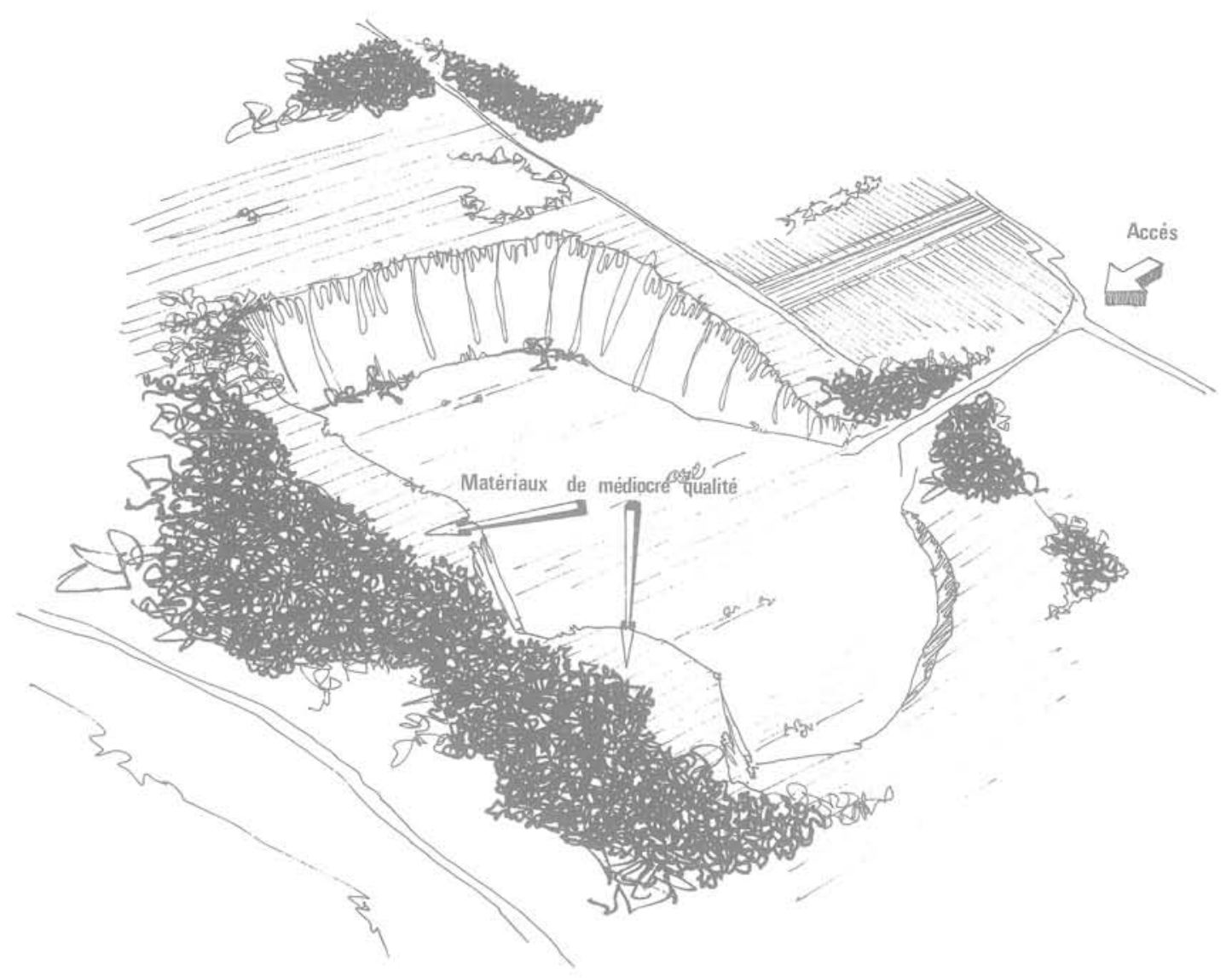

Fig. 3 - Prise en compte des hétérogénéités du matériau permettant de conserver au site une partie de sa végétation et d'isoler la carrière du milieu environnant (vue, bruit, poussières) .

Le choix du point d'ouverture d'un gisement est toujours très important, tant sur le plan économique que sur celui de l'intécration dans l'environnement; ce choix dépend de nombreux facteurs, parmi lesquels la répartition des hétérogénéités du matériau en place et l'état de fracturation du massif. Il s'agit en effet de faire en sorte que l'on soit dans les meilleures conditions techniques et économiques possibles pour la réalisation des abattages.

Par ailleurs, la connaissance de la fracturation est indispensable à l'évaluation des vibrations : il n'est pas suffisant d'écrire dans une étude d'impact que l'on prendra toutes dispositions utiles pour les minimiser, par exemple en utilisant des microretards. Dans de nombreux cas, le problème devra être examiné de beaucoup plus près, en particulier si l'exploitation prévue se trouve ầ proximité de sites sensibles, tels que blocs sur pentes instables, tunnels ferroviaires, ou tout simplement habitations ou monuments.
Ajoutons enfin que de l'état de fracturation du massif, ainsi que du pendage des couches, dépendra l'état des talus :

- en premier lieu, sous l'angle de la sécurité,

- en second lieu, sous l'angle paysager, bien que ce point de vue soit par définition discutable,

- enfin, sous l'angle de la revégétalisation: de la fracturation dépend en grande partie la possibilité ou non de réaliser des banquettes ou des gradins, et aussi d'y prévoir la plantation de tel ou tel type de végétation.

Signalons enfin que les circulations d'eau, dont nous parlerons ci-après, sont directement liées à la fracturation. 


\section{POLLUTIONS ET HETEROGENEITES}

Nous avons déjà envisagé ci-dessus l'influence de ces deux paramètres sur le choix du point d'ouverture du gisement et de l'implantation des installations de traitement. Mais ils ont également d'autres conséquences importantes dans le domaine de l'environnement:

- Si le matériau extrait nécessite un lavage sur crible, voire un débourbage en amont, il est bien évident qu'il faut prévoir le traitement des eaux chargées en fines. Ceci entrainera généralement la mise en place de bassins de décantation, qu'il faudra dimensionner correctement, afin de recycler dans l'installation les eaux traitées et éviter les rejets en rivière. Si cette question n'est pas étudiêe avec précision dès le départ, les conséquences sur'l'environnement peuvent s'avérer catastrophiques.

- Il ne faut pas oublier non plus que les volumes correspondant aux zones très hétérogènes ou très polluées, et qu'il est impossible de traiter dans l'installation, viennent s'ajouter de fait à la découverte ; dans certains gisements, ces quantités sont loin d'être négligeables et il est important de bien les repérer dans le gisement, de façon à les intégrer correctement dans les mouvements de terre prévus au cours des différentes phases de l'exploitation.

- Dans les gisements alluvionnaires extraits sous la nappe phréatique, l'extraction ellemême entraîne un lessivage des matériaux, et on constate très fréquemment des colmatages qui sont d'autant plus rapides que le matériau en place est plus riche en fines. Cette constatation sur l'importance des nappes et l'influence que peuvent avoir les extractions nous conduit plus généralement à envisager les problèmes posés par les circulations d' eau.

\section{LES CIRCULATIONE D'EAU}

On peut se demander à quel moment se termine l'étude de gisement proprement dite et quand commence l'étude hydrogéologique qui est gẻnéralement une partie importante des études d'impact.

Un certain nombre d'informations doivent être connues, ce sont essentiellement :

- La position au substratum,

- Le niveau de la nappe phriaticue, ainsi que ses fluctuations à partir de relevés piézométriques. La connaissance de ce battement de la nappe, qui suppose une étude étalée dans le temps, est importante, non seulement en vue de l'extraction, mais aussi pour une préparation correcte de la remise en état en fin d'exploitation (ou à l'avancement). En effet, cette zone est peu propice à la revégétalisation et présente un aspect visuel souvent peu agréable. Il faut souligner que la méconnaissance du niveau phréatique peut être catastrophique dans le cas où la remise en culture du site a été prévue.

- Evaluation du débit d'eau : elle est indispensable dans de nombreux cas :

- pour le lavage des matériaux dans les installations,

- lorsque l'on souhaite extraire hors d'eau et cu'ur pompage avec rabattement de la nappe est nécessaire,

- Lorsque l'étude d'impact prévoit la remise en plan d'eau en fin d'exploitation. Il s'agit dans ce cas de savoir si les circulations d'eau seront suffisantes pour éviter une eutrophisation des eaux retenues dans l'excavation.

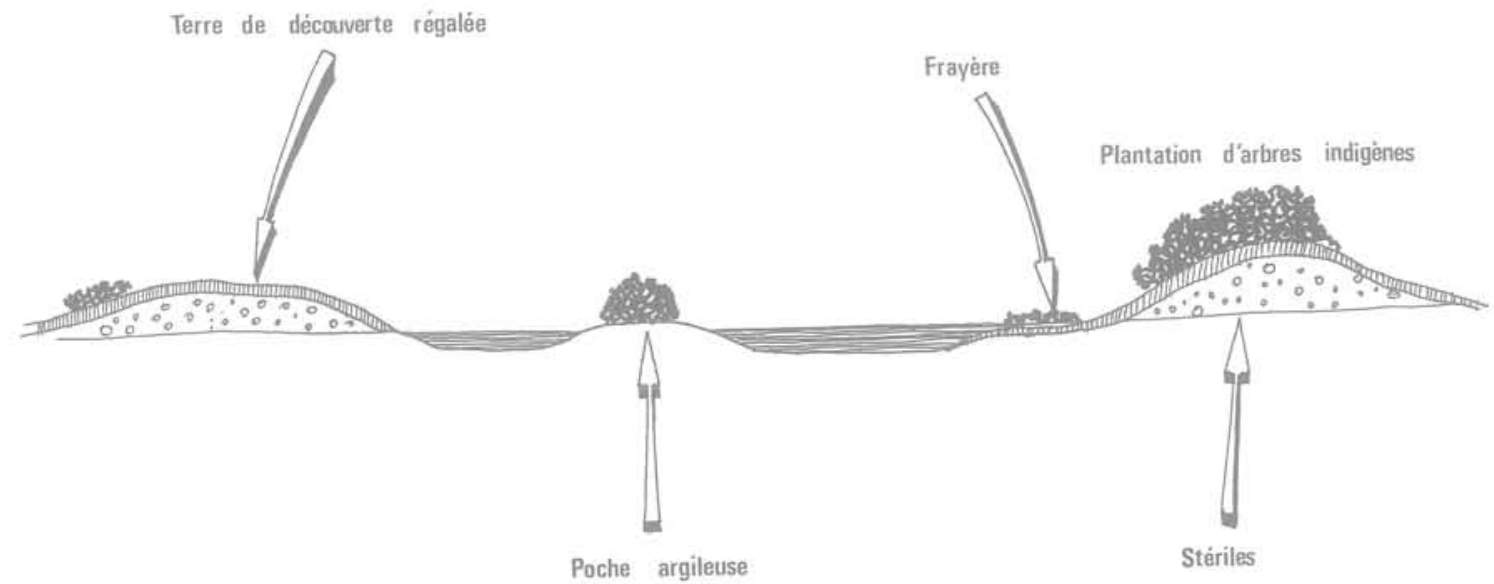

Fig. 4 - Prise en compte des paramètres d'un gisement (découverte, zones de matériau exploitable, présence de poches argileuses) pour la remise en état ultérieure. 
Toutes ces données sur les nappes concernent bien entendu surtout les matériaux alluvionnaires. Dans les zones rocheuses, le problème est souvent complexe; il faut cependant arriver à préciser ce que sera la zone d'influence de l'excavation et en particulier, les rabattements du ou des niveaux aquifères et leurs conséquences sur l'alimentation des puits du secteur. Ce problème est encore plus aigu avec les calcaires karstifiés, dans lesquels il est indispensable de se soucier des circulations prévisibles d'eaux polluées. De façon générale, les circulations d'eau en massif rocheux supposent une bonne connaissance de la structure du massif (pendage, failles, diaclases).

\section{COIJCLUS IOINS}

Les quelques points que nous venons d'énumérer concernent tantôt les gisements alluvionnaires, tantôt les gisements de roches massives, souvent les deux. Comme cet exposé ne peut et ne veut pas être exhaustif, nous n'avons pas jugé utile de les séparer. Nous avons simplement voulu montrer, à l'aide de quelques exemples, qu'il est impossible de réaliser une étude d'impact crédible si le gisement n'est pas bien connu. Il nous semEle indispensable que le paramètre "gisement" soit inclus dans les facteurs d'environnement et même qu'il soit pris en compte le premier, puisqu'il conditionne tous les autres. 